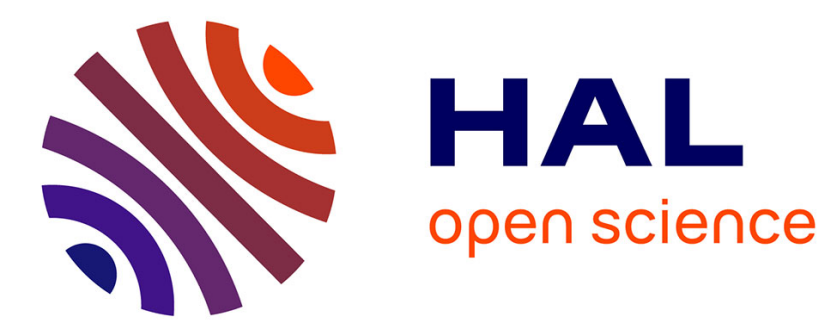

\title{
Resilience Management of Infrastructure Systems from a Multistage Decision Making Perspective
}

\author{
Yiping Fang, Enrico Zio
}

\section{To cite this version:}

Yiping Fang, Enrico Zio. Resilience Management of Infrastructure Systems from a Multistage Decision Making Perspective. 29th European Safety and Reliability Conference (ESREL2019), Sep 2019, Hannover, Germany. 10.3850/978-981-11-2724-3 . hal-02303639

\section{HAL Id: hal-02303639 \\ https://hal.science/hal-02303639}

Submitted on 2 Oct 2019

HAL is a multi-disciplinary open access archive for the deposit and dissemination of scientific research documents, whether they are published or not. The documents may come from teaching and research institutions in France or abroad, or from public or private research centers.
L'archive ouverte pluridisciplinaire HAL, est destinée au dépôt et à la diffusion de documents scientifiques de niveau recherche, publiés ou non, émanant des établissements d'enseignement et de recherche français ou étrangers, des laboratoires publics ou privés. 
Resilience Management of Infrastructure Systems from a Multistage Decision Making Perspective

\author{
Yi-Ping Fang \\ Chair on Systems Science and the Energy Challenge, Laboratoire Génie Industriel, CentraleSupélec, Université \\ Paris-Saclay, France. E-mail: viping.fang@centralesupelec.fr
}

Enrico Zio

Energy Department, Politecnico di Milano, Milano, Italy

Mines ParisTech, PSL Research University, CRC, Sophia Antipolis, France.E-mail: enrico.zio@polimi.it

The paper investigates how resilience can be understood as an operational paradigm for system management from a multistage decision making perspective. Specifically, agents involved in the resilience management aim at an optimum process of sequential preparedness/protection, emergency response, recovery, and adaptation activities, trading off the loss due to lack of system functionality with the needed investment. Agents' decisions are made sequentially aiming at minimizing the overall system loss in the presence of numerous uncertainties in the intensity of the disruption, post-disruption demand, repair durations, etc. We restrict the actions to only depend on uncertain parameters realized up to the current decision period. This process can be formulated as a multi-stage optimization problem. We discuss how the linear decision rule approximation can be used to overcome the curse of the computational complexity of the problem and to derive operationally implementable policies. By referring to a simple example of a notional infrastructure, we demonstrate that the proposed approach helps providing a holistic view of resilience management and deriving optimal actions and policies for resilience enhancement.

Keywords: Infrastructure systems, resilience management, multistage decision making.

\section{Introduction}

Resilience has emerged in the last decade as a key desired attribute of infrastructure systems (ISs, e.g., power grids, communication, transport, water distribution networks, etc.) exposed to potential disruptive events, e.g., natural hazards, accident failures or intentional attacks. The US National Academy of Science defines the concept of resilience as "the ability of a system to prepare and plan for, absorb, recover from, or more successfully adapt to actual or potential adverse events" (Cutter et al., 2013).

Many efforts have been made in the engineering community for assessing the resilience of ISs in quantitative and rigorous ways. The majority of the studies are based on either the idea of estimating the inoperability of an IS following a catastrophic event by leveraging historical event data (Nateghi, 2018; Nateghi, Guikema, \& Quiring, 2011), or the idea of predicting the time-dependent trajectory of the IS functionality under disruption through system operational models (Ouyang, Dueñas-Osorio, \& Min, 2012; Panteli \& Mancarella, 2017). Some scholars studied how to measure the resilience of interconnected ISs aiming to model the failure propagation across different systems (Fang \& Zio, 2019a, 2019b; Liu, Ferrario, \& Zio, 2017; Ouyang \& Wang, 2015; Pant, Barker, \& Zobel, 2014). Recently, fairly comprehensive surveys of the growing literature on resilience assessment have been provided for general ISs (Hosseini, Barker, \& RamirezMarquez, 2016) or specific systems like power grids (Wang, Chen, Wang, \& Baldick, 2016) and transportation (Faturechi \& Miller-Hooks, 2014; Mattsson \& Jenelius, 2015).

Despite much progress in conceptualizing and assessing resilience, significant knowledge gaps remain, especially concerning the management of IS resilience. As stated by Linkov et al. (2014) "resilience, as a property of a system, must transition from just a buzzword to an operational paradigm for system management." Alderson, Brown, and Carlyle (2015) also argued that resilience should be connected to the operational details of the system, so that proposed system changes and decisions can be naturally evaluated and actually implemented for system resilience improvement. In this sense, the science of resilience must evolve towards developing systems-level methods and tools for dealing with "unknown unknowns" in cost-efficient ways that provide a guide on how to identify and implement resilience-enhancing measures and actions.

To this end, some researchers have recently addressed the problem of supporting resilience-oriented decision-making mainly from the perspective of optimization. These studies can be categorized under two main lines: i) pre-disruption investment optimization (Alderson et al., 2015; Brown, Carlyle, Salmerón, \& Wood, 2006; Fang, Pedroni, \& Zio, 2015; Fang \& Sansavini, 2017b; Ouyang \& Fang, 2017), aiming at enhancing IS

Proceedings of the 29th European Safety and Reliability Conference.

Edited by Michael Beer and Enrico Zio

Copyright (c) 2019 European Safety and Reliability Association.

Published by Research Publishing, Singapore.

ISBN: 978-981-11-2724-3; doi:10.3850/978-981-11-2724-3_0977-cd 
resilience via optimum preventive measures, e.g. hardening and upgrading vulnerable components or deploying redundancy before a specific disruptive event strikes the system, and ii) post-event emergency response and recovery planning (Fang \& Sansavini, 2017a, 2019; Lee II, Mitchell, \& Wallace, 2007), which aims at mitigating system loss through emergency responses right after disruptions and, then, restoring a system to normal operation as quickly and efficiently as possible, e.g., through optimum resource allocation and task scheduling. However, these approaches fall short of accounting for the relations and coordination of IS resilience measures at different stages, e.g., trade-offs may exist between different stages and thus lack a holistic view of resilience management (RM).

To bridge this gap, the present paper proposes a multistage decision making perspective to address important open questions in resilience engineering. Specifically, we formulate a set of general structures of the multi-stage optimization problems under uncertainty for the RM of ISs when different uncertainty representations and risk attitudes are accounted for. Also, we discuss how the decision rule methods can be harnessed to overcome the curse of the computational complexity of the proposed problems and to derive operationally implementable policies.

The remainder of the present paper proceeds as follows. In Section 2, we discuss how the problem of RM can be modeled from a multistage decision making perspective and present two different models when different risk preferences are considered. We introduce a simple class of decision rules (DRs), i.e., the linear decision rules (LDRs), in Section 3. An exemplary problem is presented and studied in Section 4, and the conclusions are provided in Section 5.

\section{RM of ISs}

Resilience of ISs concerns a dynamic process which is the outcome of a complicated interplay between the physical changes, due to damages induced by the disruptive event, and the decisions of a mix of humans (e.g., owners, operators, managers) and autonomous "agents" (e.g., monitoring systems, feedback controllers). Henceforth, we refer to this collective decision-making entity as the "decision maker (DM)" of the IS.

IS RM can be regarded as a process of exploiting the DM's decisions, usually via coordinated and economical applications of resources, to enhance the resilience of the IS of interest. Table 1 presents some typical IS resilience-enhancement actions/strategies, associated with their times (when the action is made) and effects (how it helps to enhance resilience). It can be seen that the strategies can be either ex-ante, ex-post or even during the occurrence of a disruptive event. They make resilience improvement possible by aiding failure mitigation, or accelerating the recovery process, or the combination of the two. For example, deploying distributed backup generators in power systems makes it possible to maintain some basic level of local electricity service in the event of a bulk supply failure or emergency. Moreover, these distributed generators may also be used to re-energize the bulk network and, thus, speed up the system recovery process (Gholami, Aminifar, \& Shahidehpour, 2016). Given that the budget is always limited in practice, the most cost-effective approach requires optimum planning of the resilience actions/strategies, and trade-offs to balance current investment in failure mitigation with spending needed for recovery and adaptive capacity. Moreover, the consequences of the decisions on the resilience actions usually extend well into the future and, thus, inevitably their outcomes are affected by significant uncertainty: types and characteristics of disruptive events, levels of system damages, available resources for system restoration and unforeseen delays in repair tasks all have a bearing on the suitability of these decisions.

Table 1. Examples of actions/strategies for resilience enhancement.

\begin{tabular}{|c|c|c|c|}
\hline \multirow{2}{*}{ Actions/Strategies } & \multirow{2}{*}{ Stage } & \multicolumn{2}{|c|}{ Effect } \\
\hline & & Failure mitigation & Recovery acceleration \\
\hline Identify and protect/reinforce key components & & $\checkmark$ & \\
\hline Deploy spares (backup subsystems) & & $\checkmark$ & $\checkmark$ \\
\hline $\begin{array}{l}\text { Deploy advanced technology for system monitoring and } \\
\text { assessment }\end{array}$ & Ex-ante & $\checkmark$ & $\checkmark$ \\
\hline Preposition repair resources & & & $\checkmark$ \\
\hline Train staff and managers to increase risk awareness & & $\checkmark$ & $\checkmark$ \\
\hline $\begin{array}{l}\text { Emergency response (e.g., failure isolation, structure } \\
\text { modification) }\end{array}$ & During & $\checkmark$ & \\
\hline $\begin{array}{l}\text { Optimally schedule the repair tasks and allocate repair } \\
\text { resources }\end{array}$ & Ex-post & & $\checkmark$ \\
\hline
\end{tabular}




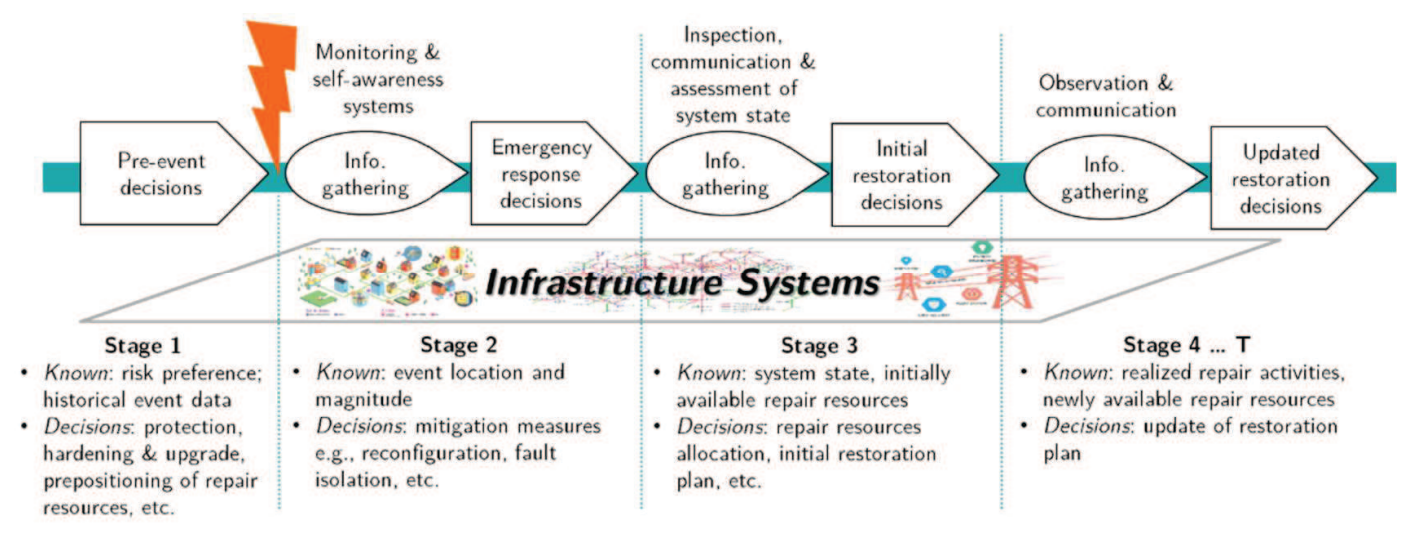

Fig. 1. Timeline indicating the flow of information and content of actions taken in RM of ISs

\subsection{RM as a Problem of Multistage Decision Making under Uncertainty}

Fig. 1 illustrates a typical timeline of flow of information and content of actions taken in RM of ISs potentially exposed to extreme disruptive events. All the features have been included in a general framework of multistage decision making under uncertainty:

Stage 1: Before the occurrence of a disruptive event, the DM makes decisions on investment in ex-ante resilience actions/strategies, such as those listed in Table 1, knowing his/her own risk preference (e.g., risk-aversion or risk-neutral) and maybe historical data about specific types of events (e.g., earthquakes). Decisions in this stage are usually referred to as here-and-now and they have to be made under multiple uncertainties about future stages.

Stage 2: During or right after the occurrence of the event and given that some information about the event (e.g., location and magnitude) is known (e.g., through monitoring, self-awareness and communication technologies), emergency response actions like system reconfiguration and failure isolation might be implemented for failure mitigation.

Stage 3: When the system is no longer impacted by the event and enters into a stable (disrupted) state, inspection and damage assessment can be conducted to support the DM decisions on how to restore the system, e.g., to select an initial restoration plan.

Stage 4...T: The initial restoration plan might be adjusted stage-wise whenever some uncertain sources are realized, e.g., the finished repair tasks take longer time than expected or new repair resources are available.
In some cases, interventions might not be possible in some stages. In a multistage decision making setting, the uncertainty sources are revealed gradually over time. Nonanticipativity of the DM's actions requires that the decisions made at the beginning of stage $i$ depend only on the information on uncertain parameters realized up to the beginning of stage $i$. In other words, the DM has no crystal ball that can reveal any deterministic information from the future.

In the following section, we present in details the mathematical formulation of the multistage decision making problems.

\subsection{Mathematical Formulation}

A DM first observes an uncertain parameter $\xi_{1}$ and, then, makes a decision $x_{1}\left(\xi_{1}\right)$ from a set of feasible decisions $X_{1}$. Subsequently, a second uncertain parameter $\xi_{2}$ is revealed, in response to which the DM takes a second decision $x_{2}\left(\xi_{1}, \xi_{2}\right) \in X_{2}$. This sequence of alternating observations and decisions extends over $T$ stage, where at any stage $t=1, \ldots, T$ the DM observes an uncertain parameter $\xi_{t}$ and selects a decision $x_{t}\left(\xi_{1}, \ldots, \xi_{t}\right) \in X_{t}$. Note that $\xi_{t}$ and $x_{t}$ are both vectors and, thus, they can represent multiple sources of uncertainty and different actions, respectively. Besides, we can simply set $\xi_{1}=1$ for deterministic here-and-now decisions when no uncertainty is observed at the first stage. To simplify the notation, we define the history of the uncertainty realization up to stage $t$ as $\xi_{[t]}=$ $\left(\xi_{1}, \ldots, \xi_{t}\right)$ and the history of selected decisions up to stage $t$ as $x_{[t]}=\left(x_{1}, \ldots, x_{t}\right)$. Moreover, we let $\xi=\left(\xi_{1}, \ldots, \xi_{T}\right)$ and $x=\left(x_{1}, \ldots, x_{T}\right)$ denote the vector concatenation of all uncertain parameters and all decisions variables, respectively.

For an IS of interest, let $f_{t}\left(x_{[t]}, \xi_{[t]}\right)$ denote a function that quantifies its performance at stage $t$ that results from all the decision choices and uncertainty realizations up to $t$. Then, the overall system performance in the studied horizon 
$F(x, \xi)$ can be measured based on a sequence of performance functions $F_{t}\left(x_{[t]}, \xi_{[t]}\right), t=1, \ldots, T$. In the present paper, we simply assume

$$
F(x, \xi)=\sum_{t=1}^{T} F_{t}\left(x_{[t]}, \xi_{[t]}\right)
$$

Then, the DM's goal in RM is to select the functions or decision rules (DRs) $x_{1}(\cdot), \ldots, x_{T}(\cdot)$, which map observation histories to decisions, such that the overall system performance $F(x, \xi)$ is maximized while all the decisions are feasible. The problem is represented as follows.

$$
\begin{array}{r}
\max F(x, \xi)=\sum_{t=1}^{T} F_{t}\left(x_{[t]}, \xi_{[t]}\right) \\
\text { s.t. } x_{t}\left(\xi_{[t]}\right) \in X_{t}, \forall \xi \in \Xi, t=1, \ldots, T
\end{array}
$$

where $\Xi$ denotes the set of all possible uncertain scenarios. Typically $\Xi$ is infinite and problem $\mathcal{P}$ accommodates infinitely many decision variables. Moreover, the objective $F(x, \xi)$ relies on the realization of uncertain scenario $\xi$ and, thus, is inherently stochastic.

Below, we present in details the formulations of more tractable versions of problem $\mathcal{P}$ by taking into account the DM's risk preferences, i.e., riskneutral or risk-aversion.

\subsubsection{Stochastic Program}

If the distribution governing the uncertainty $\xi$ is known and the DM is risk-neutral, then it is possible and reasonable to maximize the expected value of the overall system performance. Thus, problem $\mathcal{P}$ converts to the following multistage stochastic program.

$$
\begin{gathered}
\max \mathbb{E}_{\xi}\left(\sum_{t=1}^{T} F_{t}\left(x_{[t]}, \xi_{[t]}\right)\right) \\
\text { s.t. } x_{t}\left(\xi_{[t]}\right) \in X_{t}, \forall \xi \in \Xi, t=1, \ldots, T
\end{gathered}
$$

where $\mathbb{E}_{\xi}(\cdot)$ denotes expectation with respect to the random parameter $\xi$.

For its computational tractability, problem $\mathcal{S P}$ is typically simplified into a deterministic structure by reducing the uncertainty set $\Xi$ to a finite subset of suitable size through scenario sampling and reduction techniques (Dupačová, GröweKuska, \& Römisch, 2003; Fang \& Sansavini, 2019).

\subsubsection{Robust Optimization Problem}

In practice, the expected value is often a poor choice of measure for risk-informed decisions particularly for ISs resilience against catastrophic events (Alderson et al., 2015). In other words, risk-aversion is a more advocated attitude in IS RM. Moreover, the uncertainty $\xi$ may be simply unknown to the DM. In these situations, the DM may want to consider the worst-case system performance, where the worst case (minimum performance) is evaluated with respect to all possible scenarios $\xi \in \Xi$. This results in the following formulation of a general multistage (adaptive) robust optimization.

$$
\begin{gathered}
\max \min _{\xi \in \Xi}\left(\sum_{t=1}^{T} F_{t}\left(x_{[t]}, \xi_{[t]}\right)\right) \\
\text { s.t. } x_{t}\left(\xi_{[t]}\right) \in X_{t}, \forall \xi \in \Xi, t=1, \ldots, T
\end{gathered}
$$

Practically solving problem $\mathcal{R P}$ (and $\mathcal{S P}$ ) requires explicit expressions of $F_{t}\left(x_{[t]}, \xi_{[t]}\right), X_{t}$ and $\Xi$, which all depend on the specific IS of interest. In Section 4, we will demonstrate how to apply the $\mathcal{R P}$ model to a notional commodity distribution network.

\section{The Linear Decision Rule Approximation}

The decision rules, or implementable policies, are the functions $x_{1}(\cdot), \ldots, x_{T}(\cdot)$ that map (historical) uncertainties data $\xi_{[t]}$ to decisions. The concept of DRs was proposed to alleviate the curse of dimensionality of multistage stochastic programs (Georghiou, Wiesemann, \& Kuhn, 2015; Kuhn, Wiesemann, \& Georghiou, 2011). It restricts the admissible recourse decisions to some specific function forms, e.g., linear, segregated linear, piecewise linear and algebraic as well as trigonometric polynomial, of the observed uncertain parameter values to obtain a good approximation to the optimal solution of the multistage problem (Georghiou, Kuhn, \& Wiesemann, 2018). Such an approach is in line with Simon's view that human decision agents may tend to rely on heuristic rules to achieve a stated satisfactory level of performance when operating under complex and uncertain environments (Simon, 1972). Also, with DRs there is no need for dynamic re-optimization during the time-sensitive planning horizon, particularly during the emergency response and system restoration phases. Moreover, a generic DR represented by simple functions has an advantage in that they are practical and intuitive for DMs to use in operation. As such, the present paper advocates the usage of DR approaches for handling the computational complexity of multistage stochastic and robust optimization models.

LDRs are a simple but effective class of DRs $x_{t}(\cdot), t=1, \ldots, T$ that are linearly dependent on the observed uncertainty history $\xi_{[t]}$. LDRs have been proven to optimally solve the linear quadratic regulator problem, a class of one-dimensional robust control problems, and two stage robust vehicle routing problems (Georghiou et al., 2018). The LDRs can be expressed as

$$
x_{t}\left(\xi_{[t]}\right)=L_{t} \xi_{[t]}
$$

for some matrix $L_{t}$ with proper dimensions. Then, substituting these LDRs into problems $\mathcal{S P}$ and 
$\mathcal{R P}$ yields their approximate problems that are amenable to numerical solutions. The forms of the solutions are also easy to use in operations since they are linear combinations of the observed uncertainty realizations.

\section{Notional Example}

This section presents a simple example involving a notional IS adopted from Alderson et al. (2015), which is designed to distribute some commodity to different locations within an urban area. As shown in Fig. 2, there are 2 suppliers (black circles) and 14 demand locations (white circles) in this distribution network. Commodity flow is carried by links that are bidirectional and have a limited capacity. Assume that the demand at each demand location is 1 , that the supply at each supply is 10 and that each link has a flow capacity of 5 . Assume the operator faces a penalty of $€ 10$ per flow unit and per hour for each node that does not receive its demand and a per-unit transmit cost of $€ 1 /$ hour for each link. Then, the objective of the $\mathrm{DM}$ for the system is to route the available flows in order to minimize the sum of all the transmission costs and penalty costs for the system.

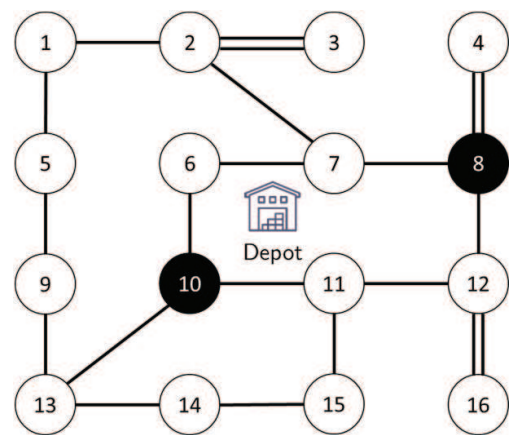

Fig. 2. A notional distribution network

The DM's task is complicated by the fact that the capacities of one or more links in the system can be damaged by disruptive events. To improve the system resilience, the DM's (pre-event) measures include i) protecting a link so that it is invulnerable to loss, and ii) prepositioning certain amount of repair resources at a depot so that they can be used for the restoration of affected links in the system (see Fig. 2). After the occurrence of a failure event, the DM assigns the available repair resources to the affected links in an optimum way so that the system performance (the minimum cost of commodity flow) is recovered as soon as possible. For simplicity, we assume that the recovery speeds of damaged links depend linearly on the allocated resources. By adopting a riskaversion attitude and considering the worst case disruption, the integrated RM problem for this notional infrastructure system is formally formulated as an $\mathcal{R P}$ in Section 4.1.

\subsection{Problem formulation}

$$
\begin{gathered}
\min _{x, y, L_{e}} \max _{\xi \in \Xi} \mathcal{H}(x, y, \xi) \\
\text { s.t. } \sum_{e \in E} c^{p} x_{e}+c^{\text {depot }} y \leq B \\
x_{e} \in\{0,1\}, y \geq 0 \\
\sum_{e \in E} L_{e} \xi \leq y, \forall \xi \\
\Xi=\left\{\xi \in[0,1]^{|E|}: \sum_{e \in E}\left(1-\xi_{e}\right) \leq \Gamma\right\} \\
\mathcal{H}(x, y, \xi)=\min _{s, f, \Delta d \in \mathcal{O}}\left(\sum_{t=1}^{T} \sum_{e \in E} 1 .\right. \\
\left.f_{e t}+\sum_{i \in V} 10 \cdot \Delta d_{i t}\right) \\
\mathcal{O}=\left\{[s, f, \Delta d] \mid 0 \leq \Delta d_{i t} \leq \hat{d}_{i}, \forall i, t\right. \\
\quad 0 \leq s_{i t} \leq \hat{s}_{i}, \forall i, t \\
s_{i t}-\sum_{e \in E \mid o(l)=i} f_{e t}+ \\
\sum_{e \in E \mid d(l)=i} f_{e t}=\hat{d}_{i}-\Delta d_{i t}, \forall i, t \\
\left|f_{e t}\right| \leq \hat{f}_{e} x_{e}+\hat{f}_{e}\left(\xi_{e}+\rho \cdot L_{e} \xi \cdot t\right)(1 \\
\left.\left|f_{e t}\right| \leq \hat{f}_{e}, \forall e, t\right\}
\end{gathered}
$$

The objective of the RM problem (3) is to minimize the worst-case system cost $\mathcal{H}(x, y, \xi)$ under all possible failure scenarios by investing in link protection $x_{e}$, repair resources prepositioning $y$ and assignment $L_{e} \xi$. It is noted that here $L_{e} \xi$ represents the amount of repair resources assigned to link $e$. It is an adjustable decision variable and is modeled through the LDRs introduced in Section 3. Constraint (4) sets the total investment budget $B$. Constraint (5) imposes the nature and lower bound of decision variables $x_{e}$ and $y$. Constraint (6) puts the limit for the total amount of assigned repair resources. The uncertainty set of system failure scenarios is given by (7), where $\xi_{e}$ represents the residual capacity [\%] of link $e$ and the parameter $\Gamma$ controls the magnitude of the failure. (8)-(13) represents the operational model of the system after a disruption. Specifically, constraints (9) and (10) impose the upper limits of unmet demand $\Delta d_{i t}$ and supply $s_{i t}$, respectively. Constraint (11) represents the flow balance at each node. Constraints (12) and (13) enforce the capacity limit for each link. Importantly, constraint (12) states that if a link is protected $x_{e}=1$ and its capacity will always be its original level $\hat{f}_{e}$; otherwise, its capacity depends on its residual capacity $\xi_{e}$ in the failure scenario and the repair speed $\rho \cdot L_{e} \xi$, where $\rho$ is the repair rate of link capacity, i.e., the percentage of capacity that can be restored per unit of repair resources and per repair time period. 
With the LDRs approach, the formulated problem (3)-(13) is relatively tractable compared to its multistage counterpart (Georghiou et al., 2015) and can be solved using state-of-the-art MIP solvers like CPLEX.

\subsection{Numerical Results}

Fig. 3 shows the results of the RM objective, i.e., the cumulative system cost during the failure horizon, under different values of investment budget $B$ and failure magnitude $\Gamma$ for a repair rate $\rho=$ 0.2 . It is clearly shown that the system cost grows monotonically with the increase of the failure magnitude, which, fortunately, can be mitigated by increasing the investments in link protection and repair resource prepositioning. With each additional investment in these resilience measures, the system cost vs. failure magnitude $\Gamma$ curve becomes less steep, indicating improved operational resilience for the system.

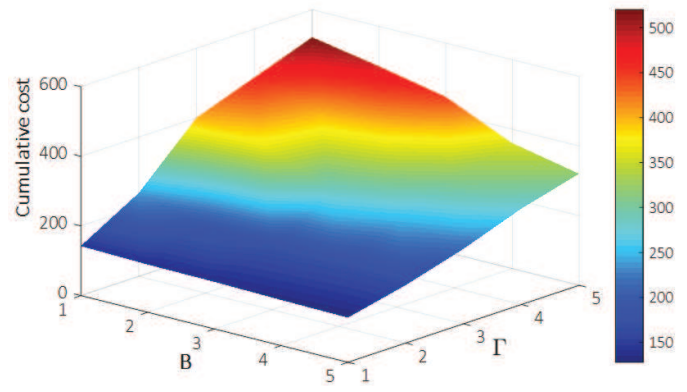

Fig. 3. Cumulative system cost under different values of investment budget $B$ and failure magnitude $\Gamma$ for $\rho=0.2$
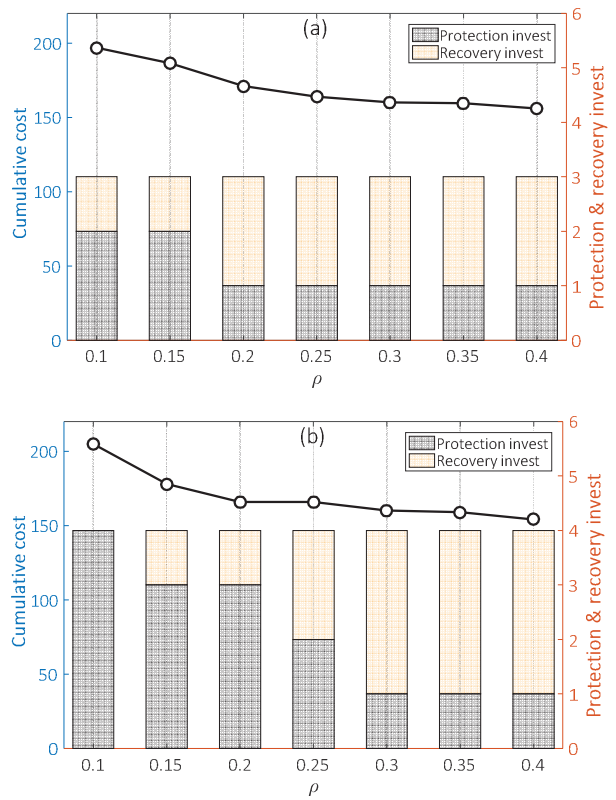
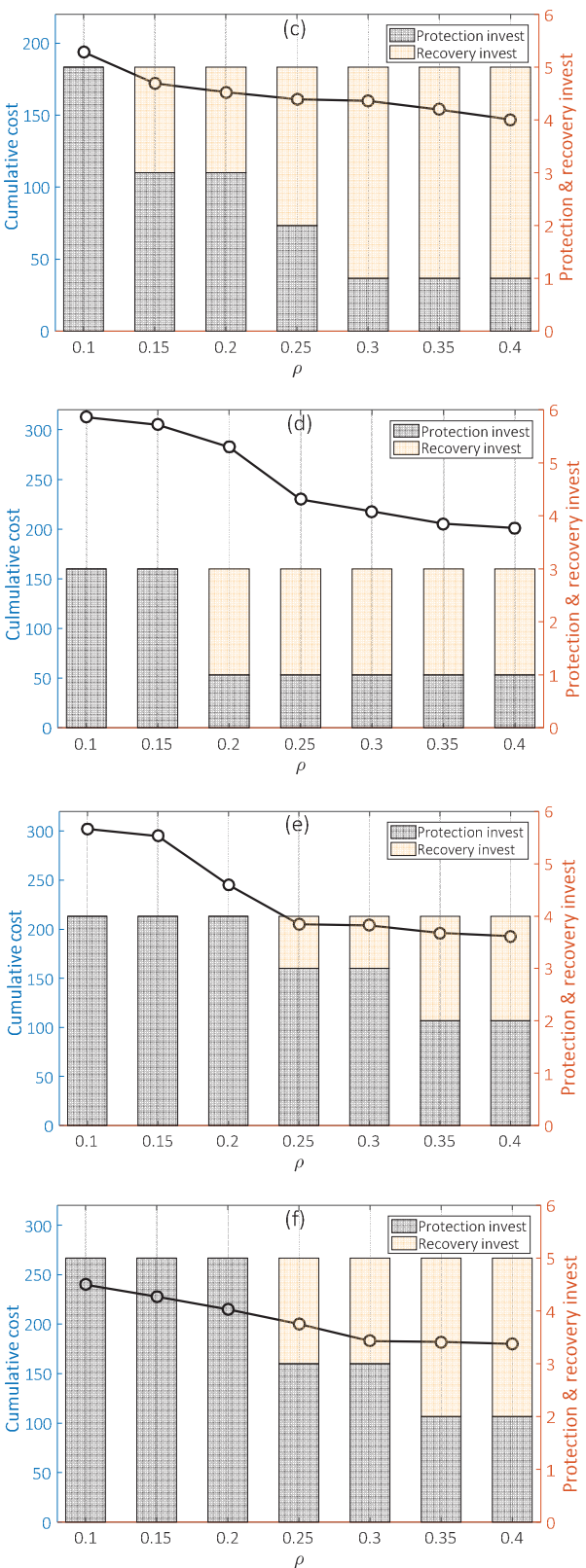

Fig. 4. Cumulative system cost and protection \& recovery investment under different values of link repair rate $\rho$ for (a) $B=3, \Gamma=2$, (b) $B=4, \Gamma=2$, (c) $B=5, \Gamma=2$, (d) $B=$ 3, $\Gamma=3$, (e) $B=4, \Gamma=3$, and (f) $B=5, \Gamma=3$

Fig. 4(a)-(f) show the results of the cumulative system costs, the investments in link protection (protection invest) and the investments in prepositioning repair resources (recovery invest) along with different values of link repair rate $\rho$ for different cases of $B$ and $\Gamma$. As expected, high values of the link repair rate $\rho$ generally result in reduced cumulative system costs in all the cases (a)-(f), 
representing more resilient infrastructure systems. Moreover, it can be observed in Fig. 4(a)-(f) that the optimum solutions tend to make more investments in protecting critical links than in prepositioning repair resources for small values of the repair rate $\rho$; when $\rho$ increases, the optimum solutions shift to invest more in placing repair resources in the depot while reducing the protection investment (the total investment keeps unchanged for each case). In other words, clear trade-offs exist between the expenditure of pre-event link protection and the investment in repair resources preparation for different values of link repair rate.

\section{Conclusions}

Resilience needs to develop towards an operational paradigm for system management. In this view, the present paper proposes a multistage decision making perspective on resilience management problems. By taking into account different uncertainty representations and risk attitudes, a set of general structures of the multi-stage optimization problems (i.e., $\mathcal{P} \mathcal{S P}, \mathcal{R P}$ ) have been formulated for the resilience management of infrastructure systems.

Although the formulated problems are usually very challenging to be solved, the general DR approaches can be used to alleviate the computational burden. A generic DR represented by simple functions is practical and intuitive for DMs to use in operation. Also, by using DRs, there is no need for dynamic re-optimization during the timesensitive emergency response and system restoration phases. In the present paper, we introduce the simplest but effective LDRs and demonstrates its effectiveness through a simple infrastructure case study.

To demonstrate the application of our proposed framework, along with supporting analytic techniques, a simple example of a notional infrastructure has been considered. We illustrate how the resilience improvement problem for the system can be formulated as a multistage robust optimization by adopting a risk-aversion attitude and considering the worst case failure scenarios. The results clearly show the trade-offs and coordination of the resilience decisions at different stages.

\section{References}

Alderson, D. L., Brown, G. G., \& Carlyle, W. M. (2015). Operational models of infrastructure resilience. Risk Analysis, 35(4), 562-586.

Brown, G., Carlyle, M., Salmerón, J., \& Wood, K. (2006). Defending critical infrastructure. Interfaces, 36(6), 530-544.

Cutter, S. L., Ahearn, J. A., Amadei, B., Crawford, P., Eide, E. A., Galloway, G. E., . . . SchochSpana, M. (2013). Disaster resilience: A national imperative. Environment: Science and
Policy for Sustainable Development, 55(2), 25-29.

Dupačová, J., Gröwe-Kuska, N., \& Römisch, W. (2003). Scenario reduction in stochastic programming. Mathematical programming, 95(3), 493-511.

Fang, Y.-P., Pedroni, N., \& Zio, E. (2015). Optimization of cascade-resilient electrical infrastructures and its validation by power flow modeling. Risk Analysis, 35(4), 594-607.

Fang, Y.-P., \& Sansavini, G. (2017a). Emergence of antifragility by optimum postdisruption restoration planning of infrastructure networks. Journal of Infrastructure Systems, 23(4), 04017024.

Fang, Y.-P., \& Sansavini, G. (2017b). Optimizing power system investments and resilience against attacks. Reliability Engineering \& System Safety, 159, 161-173.

Fang, Y.-P., \& Sansavini, G. (2019). Optimum post-disruption restoration under uncertainty for enhancing critical infrastructure resilience. Reliability Engineering \& System Safety, 185, $1-11$.

Fang, Y.-P., \& Zio, E. (2019a). An Adaptive Robust Framework for the Optimization of the Resilience of Interdependent Infrastructures under Natural Hazards. European Journal of Operational Research.

Fang, Y.-P., \& Zio, E. (2019b). Game-Theoretic Decision Making for the Resilience of Interdependent Infrastructures Exposed to Disruptions Critical Infrastructure Security and Resilience (pp. 97-114): Springer.

Faturechi, R., \& Miller-Hooks, E. (2014). Measuring the performance of transportation infrastructure systems in disasters: A comprehensive review. Journal of infrastructure systems, 21(1), 04014025.

Georghiou, A., Kuhn, D., \& Wiesemann, W. (2018). The decision rule approach to optimization under uncertainty: methodology and applications. Computational Management Science, 1-32.

Georghiou, A., Wiesemann, W., \& Kuhn, D. (2015). Generalized decision rule approximations for stochastic programming via liftings. Mathematical Programming, 152(1-2), 301338.

Gholami, A., Aminifar, F., \& Shahidehpour, M. (2016). Front lines against the darkness: Enhancing the resilience of the electricity grid through microgrid facilities. IEEE Electrification Magazine, 4(1), 18-24.

Hosseini, S., Barker, K., \& Ramirez-Marquez, J. E. (2016). A review of definitions and measures of system resilience. Reliability Engineering \& System Safety, 145, 47-61.

Kuhn, D., Wiesemann, W., \& Georghiou, A. (2011). Primal and dual linear decision rules in 
stochastic and robust optimization. Mathematical Programming, 130(1), 177-209.

Lee II, E. E., Mitchell, J. E., \& Wallace, W. A. (2007). Restoration of services in interdependent infrastructure systems: A network flows approach. IEEE Transactions on Systems, Man, and Cybernetics, Part C (Applications and Reviews), 37(6), 1303-1317.

Linkov, I., Bridges, T., Creutzig, F., Decker, J., Fox-Lent, C., Kröger, W., . . . Nathwani, J. (2014). Changing the resilience paradigm. Nature Climate Change, 4(6), 407.

Liu, X., Ferrario, E., \& Zio, E. (2017). Resilience analysis framework for interconnected critical infrastructures. ASCE-ASME Journal of Risk and Uncertainty in Engineering Systems, Part B: Mechanical Engineering, 3(2), 021001.

Mattsson, L.-G., \& Jenelius, E. (2015). Vulnerability and resilience of transport systems-A discussion of recent research. Transportation Research Part A: Policy and Practice, 81, 16-34.

Nateghi, R. (2018). Multi-dimensional infrastructure resilience modeling: an application to hurricane-prone electric power distribution systems. IEEE Access, 6, 13478-13489.

Nateghi, R., Guikema, S. D., \& Quiring, S. M. (2011). Comparison and validation of statistical methods for predicting power outage durations in the event of hurricanes. Risk Analysis: An International Journal, 31(12), 1897-1906.

Ouyang, M., Dueñas-Osorio, L., \& Min, X. (2012). A three-stage resilience analysis framework for urban infrastructure systems. Structural safety, 36, 23-31.

Ouyang, M., \& Fang, Y. (2017). A mathematical framework to optimize critical infrastructure resilience against intentional attacks. Computer-Aided Civil and Infrastructure Engineering, 32(11), 909-929.

Ouyang, M., \& Wang, Z. (2015). Resilience assessment of interdependent infrastructure systems: With a focus on joint restoration modeling and analysis. Reliability Engineering \& System Safety, 141, 74-82.

Pant, R., Barker, K., \& Zobel, C. W. (2014). Static and dynamic metrics of economic resilience for interdependent infrastructure and industry sectors. Reliability Engineering \& System Safety, 125, 92-102.

Panteli, M., \& Mancarella, P. (2017). Modeling and evaluating the resilience of critical electrical power infrastructure to extreme weather events. IEEE Systems Journal, 11(3), 1733-1742.

Simon, H. A. (1972). Theories of bounded rationality. Decision and organization, 1(1), 161176.

Wang, Y., Chen, C., Wang, J., \& Baldick, R. (2016). Research on resilience of power systems under natural disasters-A review.
IEEE Transactions on Power Systems, 31(2), 1604-1613. 